\title{
SEARCHING FOR PLANETS IN HOLEY DEBRIS DISKS WITH THE APODIZING PHASE PLATE*
}

\author{
Tiffany Meshiat ${ }^{1}$, Vanessa P. Bailey ${ }^{2}$, Kate Y. L. Su ${ }^{2}$, Matthew A. Kenworthy ${ }^{1}$, \\ Eric E. MamajeK ${ }^{3}$, Phillip M. Hinz ${ }^{2}$, and Paul S. SMith ${ }^{2}$ \\ ${ }^{1}$ Sterrewacht Leiden, P.O. Box 9513, Niels Bohrweg 2, 2300 RA Leiden, The Netherlands \\ 2 Steward Observatory, Department of Astronomy, University of Arizona, 933 North Cherry Avenue, Tucson, AZ 85721-0065, USA \\ ${ }^{3}$ Department of Physics and Astronomy, University of Rochester, Rochester, NY 14627-0171, USA \\ Received 2014 October 1; accepted 2014 December 4; published 2015 February 2
}

\begin{abstract}
We present our first results from a high-contrast imaging search for planetary mass companions around stars with gapped debris disks, as inferred from the stars' bright infrared excesses. For the six considered stars, we model the disks' unresolved infrared spectral energy distributions in order to derive the temperature and location of the disk components. With VLT/NaCo Apodizing Phase Plate coronagraphic $L^{\prime}$-band imaging, we search for planetary mass companions that may be sculpting the disks. We detect neither disks nor companions in this sample, confirmed by comparing plausible point sources with archival data. In order to calculate our mass sensitivity limit, we revisit the stellar age estimates. One target, HD 17848, at 540 \pm 100 Myr old is significantly older than previously estimated. We then discuss our high-contrast imaging results with respect to the disk properties.
\end{abstract}

Key words: circumstellar matter - planetary systems - stars: individual (HD 17848, HD 28355, HD 37484, HD 95086)

\section{INTRODUCTION}

Despite the large number of direct imaging surveys (SEEDS: Brandt et al. 2014; Janson et al. 2013, NICI: Biller et al. 2013; Nielsen et al. 2013; Wahhaj et al. 2013, GDPS: Lafrenière et al. 2007, IDPS: Vigan et al. 2012, and with NaCo: Desidera et al. 2014; Chauvin et al. 2014) and the hundreds of sources observed, few planets have been discovered from these surveys (HR8799 bcde from IDPS: Marois et al. 2008, 2010, GJ 504 b from SEEDS; Kuzuhara et al. 2013). This low detection rate is likely due to a combination of factors (e.g., the wavelength of the observations, the target selection, the lack of a dedicated exoplanet instrument, etc). The faint $H$-band detection of HD 95086 $\mathrm{b}$ (Galicher et al. 2014) and the non-detection companion candidate around HD 169142 (Reggiani et al. 2014; Biller et al. 2014) demonstrate that these low mass companions are extremely red. These results reinforce the importance of searching for planets in the thermal infrared with $L^{\prime}$-band $(3.8 \mu \mathrm{m})$, as the planetto-star contrast is reduced and contamination from background stars is strongly suppressed.

We aim to find a signpost for planets; a trait of the stars or systems which might yield a higher planet detection rate than previous surveys. One possible signpost is the structure of debris disks with gaps, which dynamically may imply the presence of a planet (Quillen \& Thorndike 2002; Quillen 2006). This includes both debris systems with a large hole in the center, generally classified as one-component disks, and debris systems with a large gap, generally classified as two-component (warm inner and cool outer) disks (Su \& Rieke 2014). We have designed the "Holey Debris Disks" exoplanet direct imaging survey guided by this hypothesis. The targets were selected based on several criteria: youth, distance, brightness, and unresolved infrared excess indicative of the presence of a possibly sculpted debris disk around the star using Spitzer data (Su et al. 2010). Our constraints on companion masses and locations will provide useful inputs for future debris disk/planet dynamical models.

\footnotetext{
* Based on observations collected at the European Organization for Astronomical Research in the Southern Hemisphere, Chile, ESO under program numbers 090.C-0148(A) and 091.C-0457(A)
}

We present the first results of the Holey Debris Disk survey, obtained at the Very Large Telescope (VLT) with the $\mathrm{NaCo}^{4}$ (Lenzen et al. 2003; Rousset et al. 2003) instrument and Apodizing Phase Plate coronagraph (APP: Kenworthy et al. 2010; Quanz et al. 2010). The remainder of the survey data, obtained with the LMIRCam ${ }^{5}$ (Skrutskie et al. 2010; Leisenring et al. 2012), Clio2 (Sivanandam et al. 2006), and NICI $^{6}$ (Chun et al. 2008) instruments, will be presented in a companion paper. Subsets of the SEEDS survey (Janson et al. 2013) and the NICI survey (Wahhaj et al. 2013) focused on similar debris disk targets at $H$-band; however, we chose to image our sample with $\mathrm{NaCo}$ in the thermal infrared ( $L^{\prime}$-band). This method does not rely on methane absorption in the planet's atmosphere (as Spectral Differential Imaging does; Marois et al. 2000), nor is it negatively impacted by the reddening effect of thick clouds. The majority of planets found to date, despite their relatively cool effective temperatures, lack methane absorption and have thick clouds (e.g., HR8799 bcd and 2MASS 1207 b Skemer et al. 2014).

The direct detection of close-in planets is limited by instrumental diffraction and scattering effects on the point-spread function (PSF) of the bright primary star. The scattered light may be much brighter than a companion. Pupil apodizing coronagraphs block the primary star's light, suppressing its PSF at the expense of throughput ( $\sim 40 \%$ suppressed for the APP: Quanz et al. 2010). We use the APP coronagraph on $\mathrm{NaCo}$ at the VLT to increase our sensitivity around our stars.

This is the first paper in a series for the Holey Debris Disks project, discussing the VLT/NaCo APP coronagraphic data and results. In Section 2 we describe the APP observations and data reduction. In Section 3 we present the methods and data used for deriving the disk properties. In Section 4 we show the resulting contrast curves for each of our targets and discuss the significance of our sensitivity with respect to the disk properties derived from disk models. We conclude in Section 5.

\footnotetext{
4 Nasmyth Adaptive Optics System (NAOS) Near-Infrared Imager and Spectrograph (CONICA).

5 L/M-band mid-InfraRed Camera.

6 Near-Infrared Coronagraphic Imager.
} 
Table 1

Observing Log for APP NaCo/VLT Runs 090.C-0148(A) and 091.C-0457(A)

\begin{tabular}{llcc}
\hline \hline Target & \multicolumn{1}{c}{$\begin{array}{c}\text { Observation Dates }{ }^{\mathrm{a}} \text { UT } \\
(\text { Hem 1, Hem 2) }\end{array}$} & $\begin{array}{c}\text { Total Integration Time } \\
(\mathrm{s})\end{array}$ & $\begin{array}{c}\text { On-sky Rotation } \\
\left({ }^{\circ}\right)\end{array}$ \\
\hline HD 28355 & 2012 Nov 17, 2013 Jan 14 & $\begin{array}{c}\text { Average DIMM Seeing } \\
\left({ }^{\prime \prime}\right)\end{array}$ & $24.80,19.56$ \\
HD 17848 & 2012 Nov 22, 2013 Jan 15 & 2580,2060 & $19.37,28.55$ \\
HD 37484 & 2013 Jan 21, 2013 Jan 25 & 1940,2860 & $27.86,10.82$ \\
HD 95086 & 2013 Apr 26, 2013 May 16 & 1360,2232 & 0.78 \\
HD 134888 & 2013 Apr 21, $\cdots$ & 3800,3120 & $24.85,20.50$ \\
HD 110058 & 2013 Apr 25, $\cdots$ & $3800, \cdots$ & $93.04, \cdots$ \\
\hline
\end{tabular}

Notes. ${ }^{\text {a }}$ Data are listed in chronological order from when the first hemisphere was observed. The last two targets were only observed in one APP hemisphere. Any difference in integration time between hemispheres was unintentional, simply due to the conditions of the night.

\section{APP OBSERVATIONS AND DATA REDUCTION}

\subsection{Observations}

The APP data were obtained in 2012 and 2013 (090.C0148(A) and 091.C-0457(A), PI: Tiffany Meshkat) at the VLT/UT4 with NaCo. The APP was used for additional diffraction suppression from 0.2 to $1^{\prime \prime} .0$, increasing the chance of detecting faint companions close to the target stars. The infrared wavefront sensor was used with the target stars themselves as the natural guide star. Data were acquired with the L27 camera (27 mas/pix) and the $L^{\prime}$-band filter $(\lambda=3.80 \mu \mathrm{m}$ and $\Delta \lambda=0.62 \mu \mathrm{m})$. We used pupil tracking mode for Angular Differential Imaging (ADI; Marois et al. 2006) and intentionally saturated the stellar PSF core to increase the signal-to-noise ratio $(\mathrm{S} / \mathrm{N})$ from possible faint companions. We also obtained unsaturated data to calibrate the photometry relative to the central star and determine the sensitivity achieved in each data set.

The APP suppresses diffraction over a $180^{\circ}$ hemisphere on one side of the target star. Thus, two data sets need to be acquired, with different initial position angles (P.A.s) for full coverage around the target star. We observed six targets with the APP (see Table 1), however only four of these have complete APP hemisphere coverage around all P.A.s. One of the targets (HD 134888) has $270^{\circ}$ coverage with only one APP hemisphere (from $135^{\circ}$ to $-135^{\circ}$ ), the final target (HD 110058) does not have sufficient coverage for a detailed analysis.

Data were obtained in cube mode. Each data cube contains 200 frames, with an integration time between $0.1 \mathrm{~s}$ to $0.3 \mathrm{~s}$ per frame, depending on the $L^{\prime}$ mag of the star. Details about total integration time and field rotation per target are contained in Table 1. A three-point dither pattern (with an amplitude of $4^{\prime \prime}$ ) was used on the detector to subtract the sky background systematics from each data set, detailed in Kenworthy et al. (2013).

\subsection{Data Reduction}

Data cubes closest in time at different dither positions were subtracted from each other and centroided. The average is taken over the subtracted cube in order to decrease the full data set size. The final averaged frames cover a rectangular area of $33^{\prime \prime} 1$ by 1 1.5 centered on the star. The reason for this asymmetry is that the APP can only observe on the upper quartile portion of the CCD, due to the wedge, deliberately introduced in the optics to avoid ghost images (Kenworthy et al. 2010). Thus, we have complete coverage around the star out to 1 1.5 and incomplete coverage beyond that radius.

Optimized principal component analysis (PCA) was run on each target APP hemisphere independently, following Meshkat et al. (2014). PCA processed frames were derotated, averaged over, and combined with the other hemisphere to generate the final image with North facing up. If two regions of APP data overlap, those regions were combined by average. We generated final PCA processed frames for a range of principal components (PCs) from 3 up to 20. This first test was to determine if there were any companion candidates in our data, and if so, how robust they were to the number of PCs used in the image reduction.

We next fixed the number of PCs (approximately 10\% of cubes in the data set). We injected artificial planets in the data cubes and ran the extraction pipeline to generate contrast curves of point sources sensitivity for $5 \sigma$. The artificial planets were generated from the data with unsaturated PSF cores. The unsaturated data was scaled to the same exposure time as the saturated data. Since the APP is in the pupil plane, it affects the PSF of every source in the field of view (FOV) in the same way, thus we can use the unsaturated star itself to generate artificial planets. The artificial planets were added to the data before PCA. The planets were added at different angular separations from $0^{\prime \prime} .18$ to $3^{\prime \prime} .0$ in steps of $0{ }^{\prime \prime} 15$ and at different contrasts from 5 to $12 \mathrm{mag}$ in steps of $1 \mathrm{mag}$. This was repeated for two different P.A.s, in order to place a fake planet in each APP hemisphere. We smoothed the final PCA image by a $1 \lambda / \mathrm{D}$ aperture (Bailey et al. 2013). We measured the $S / N$ of the injected planet and decreased the flux of the injected planet until it reached a $\mathrm{S} / \mathrm{N}$ of 5 . The $\mathrm{S} / \mathrm{N}$ was determined by dividing the flux in one pixel at the center of the injected planet by the noise in a 2 pixel wide ring around the star at the planet separation (not including the planet). We then interpolated between the contrasts to determine the $5 \sigma$ contrast limit.

\section{DEBRIS DISK SEDs AND DERIVATION OF DISK PROPERTIES}

For the five stars that have complete or nearly complete APP coverage, we derive disk properties using both Spitzer and Herschel data with spectral energy distribution (SED) models and quote the results of HD 95086 from $\mathrm{Su}$ et al. (2014). We focus on the properties of the cold component, because it is the dominant part of the debris disk SED and is also more relevant to our direct imaging observations for low-mass companions because of the inner working angle in our images.

\subsection{Spitzer and Herschel Data Reduction}

Our targets were selected based on the presence of a bright, unresolved infrared excess indicative of a dusty debris disk. Most of them have well covered SEDs using Spitzer broad-band and spectroscopic data and Herschel broad-band photometry. 

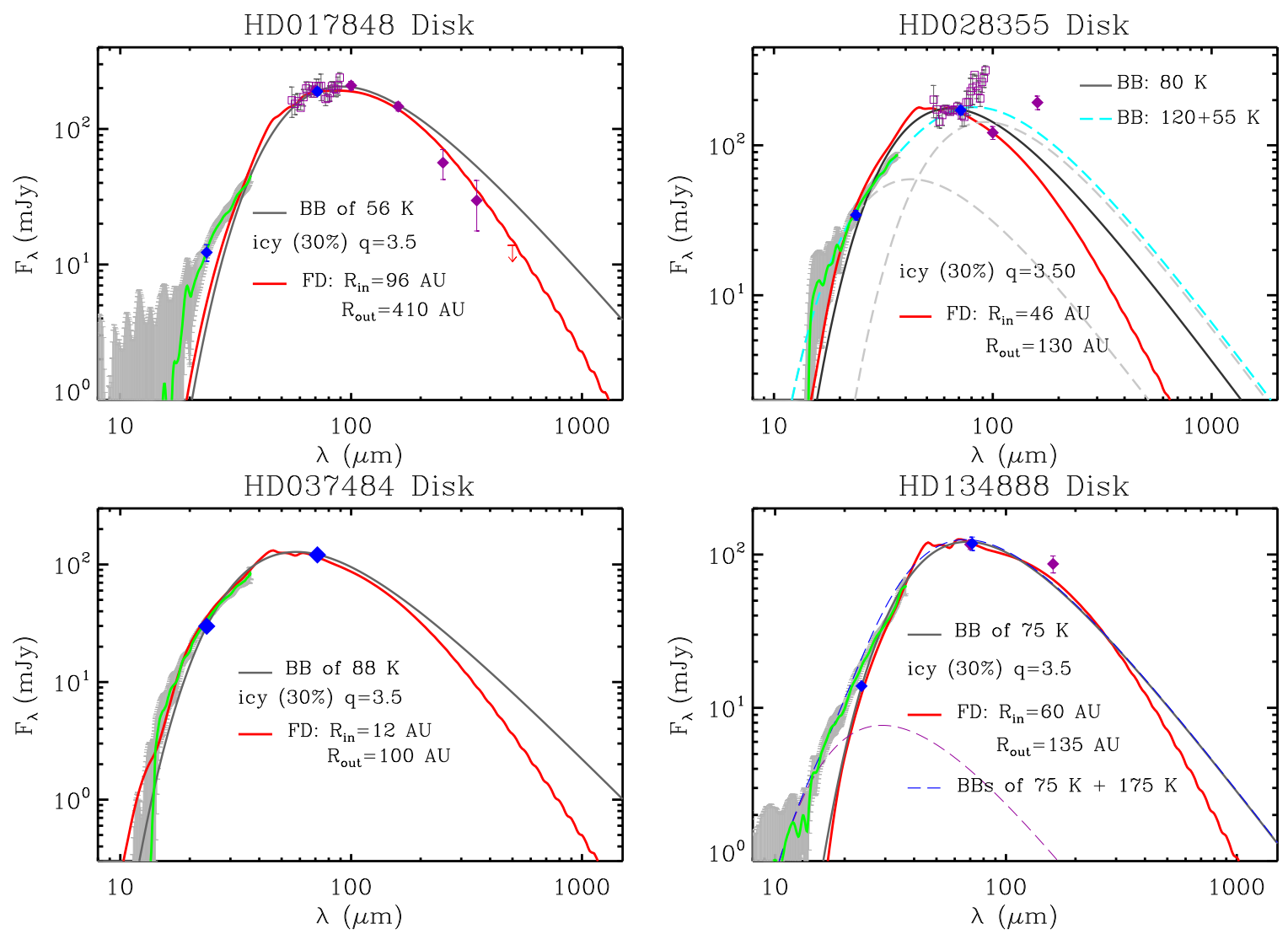

Figure 1. SEDs of the four debris systems used to derive disk properties. For all the targets, the stellar photosphere has been subtracted. In all panels, the observed data are shown in various symbols and colors: blue diamonds: Spitzer MIPS 24 and $70 \mu \mathrm{m}$ photometry; purple diamonds: Herschel PACS and SPIRE photometry; small green dots: Spitzer IRS spectrum; and purple squares: Spitzer MIPS-SED data. Various lines are SED fits: black solid lines for single blackbody emission, and red solid lines for the one-component icy grain SED model. For both the HD 28355 and HD 134888 disks, two blackbody temperature fit is also shown for comparison.

We collected all the published broad-band photometry from the literature, and performed our own photometry measurements if the data were not published. Spitzer MIPS 24 and $70 \mu \mathrm{m}$ photometry is part of the Spitzer Debris Disk Master Catalog (K. Y. L. Su et al., in preparation), where data reduction and photometry extraction were briefly summarized in Sierchio et al. (2014). Herschel PACS data reduction and photometry extraction were performed following the procedure published by Balog et al. (2014) for calibration stars, except that, as detailed in the following section, smaller apertures were used for photometry measurements to minimize background contamination. All our debris disk targets have existing Spitzer IRS low-resolution spectra. We downloaded the extracted spectra using the CASSIS database (Lebouteiller et al. 2011). Three of the targets have MIPS-SED low-resolution spectra covering 55 to $95 \mu \mathrm{m}$ with a slit width of $\sim 20^{\prime \prime}$. We reduced and calibrated the MIPS-SED data as described by Lu et al. (2008).

\subsection{Spitzer and Herschel Fluxes}

Herschel photometry of HD 95086 has been published by Moór et al. (2013) and Su et al. (2014). Here we briefly summarize the Hershel photometry results for HD 134888, HD 28355 and HD17848. The PACS 70 and $160 \mu$ m observation for HD 134888 was obtained under Program OT1_dpadget_1. The source appears to be point-like surrounded by background cirrus structure apparent on the $160 \mu \mathrm{m}$ data, therefore we used an aperture size of $6^{\prime \prime}$ at $70 \mu \mathrm{m}$ and $11^{\prime \prime}$ at $160 \mu \mathrm{m}$ to measure the photometry. Including the absolute calibration errors $(7 \%$,
Balog et al. 2014), the PACS photometry for HD 134888 is: $117 \pm 8.4 \mathrm{mJy}$ and $87 \pm 11 \mathrm{mJy}$ at 70 and $160 \mu \mathrm{m}$, respectively, and the PACS $70 \mu \mathrm{m}$ photometry agrees with the MIPS $70 \mu \mathrm{m}$ photometry $(119 \pm 12 \mathrm{mJy})$ very well.

For HD 28355, PACS 100 and $160 \mu \mathrm{m}$ data were obtained under Program OT2_fmorales_3. The source appears to be point-like, located near a bright source $\sim 28^{\prime \prime}$ away. To avoid contamination from the nearby bright source which is extended at $160 \mu \mathrm{m}$, we used an aperture size of $6^{\prime \prime}$ to measure the photometry at both bands. Our adopted photometry for the HD 28355 system is $127 \pm 12 \mathrm{mJy}$ and $195 \mathrm{mJy} \pm 20 \mathrm{mJy}$ at 100 and $160 \mu \mathrm{m}$, respectively. The contamination from the nearby bright source explains the up-turn in the MIPS-SED data for wavelengths longward of $\sim 70 \mu \mathrm{m}$ (see Figure 1).

The PACS and SPIRE data for HD 17848 were obtained under program $O T 1 \_p a b r a h a m \_2$ using all the available photometry bands. The source appears to be elongated, suggesting a close to edge-on orientation to the disk. We used an aperture size of $22^{\prime \prime}$ to measure photometry at all three bands, at which radius the encircled flux reached the maximum and flattened afterward. The final PACS fluxes are: $201 \pm 14 \mathrm{mJy}, 213 \pm 15 \mathrm{mJy}$, and $148 \pm 11 \mathrm{mJy}$ at 70,100 and $160 \mu \mathrm{m}$, respectively. The PACS $70 / 100 \mu \mathrm{m}$ photometry agrees well with the MIPS $70 \mu \mathrm{m}$ and MIPS-SED data (see Figure 1). For the SPIRE data, we used the level 2 product for point sources provided by the Herschel Science Center (HIPE ver. 11). The source was detected at $\gtrsim 3 \sigma$ at both 250 and $350 \mu \mathrm{m}$, but not detected at $500 \mu \mathrm{m}$. The submillimeter fluxes are: $57 \pm 14 \mathrm{mJy}, \quad 30 \pm 12 \mathrm{mJy}$ and $<14 \mathrm{mJy}(1 \sigma)$ at 250,350 and $500 \mu \mathrm{m}$, respectively. 
Table 2

Stellar and Disk Properties for Targets

\begin{tabular}{|c|c|c|c|c|c|c|}
\hline Target & $\begin{array}{l}\text { Distance } \\
(\mathrm{pc})\end{array}$ & $\begin{array}{c}L^{\prime} \\
(\mathrm{mag})\end{array}$ & $\begin{array}{l}\text { Age } \\
\text { (Myr) }\end{array}$ & $\begin{array}{c}\text { Cold Disk Temperature } \\
(\mathrm{K})\end{array}$ & $\begin{array}{c}\text { Cold Disk Inner/outer Edge } \\
(\mathrm{AU})\end{array}$ & $\begin{array}{l}\text { Dust Mass } \\
\left(10^{-3} M_{\oplus}\right)\end{array}$ \\
\hline HD1 7848 & $50.5 \pm 0.5$ & 5.0 & 540 & 56 & $96_{-37}^{+9}, 410_{-96}^{+24}$ & $1.3 \pm 0.7$ \\
\hline HD 28355 & $48.8 \pm 0.7$ & 4.5 & 625 & 80 & $46 \pm 12,130 \pm 30$ & $1.8 \pm 0.7$ \\
\hline HD 37484 & $56.7 \pm 2.0$ & 6.3 & 30 & 88 & $12_{-4}^{+20}, 100_{-20}^{+100}$ & $2 \pm 1$ \\
\hline HD 95086 & $90.4 \pm 3.3$ & 6.7 & 17 & 55 & $63 \pm 6,189 \pm 13$ & 200 \\
\hline HD 134888 & $89.0 \pm 8.4$ & 7.6 & 16 & 75 & $60 \pm 11,135 \pm 29$ & $32.5 \pm 14$ \\
\hline HD 110058 & $106.7 \pm 8.3$ & 7.5 & 10 & $\cdots$ & $\cdots$ & $\cdots$ \\
\hline
\end{tabular}

These values are consistent with the Herschel photometry recently published by Pawellek et al. (2014) within uncertainties.

The final disk SEDs were constructed by subtracting the best-fit Kurucz ATLAS9 stellar atmosphere models (Castelli \& Kurucz 2004) that fit the optical and near-infrared data. The uncertainties in the excess fluxes also included $2 \%$ photospheric extrapolation errors. Figure 1 shows the disk SEDs for our debris targets. We have excluded the disk SED for HD 95086, which will be published in Su et al. (2014), and for HD 110058, on which we did not obtain sufficient APP sky coverage for analysis.

\subsection{Methodology of Deriving Disk Properties}

To estimate the likely debris location, we performed basic SED modeling. We started with the simplest blackbody fitting for the disk SEDs (with a typical error of $\pm 5 \mathrm{~K}$ ) and used these temperatures to guide a more complicated SED model with adopted grain properties. Without spatial information, SED modeling is degenerate; therefore, our strategy is to minimize model parameters with some reasonable assumptions.

Similar to the SED model for HD 95086 ( $\mathrm{Su}$ et al. 2014), we adopted icy silicates as our grain properties. The particle size distribution was assumed to be a power-law form, $\sim a^{-q}$, where $a$ is the grain radius with a minimum $a_{\text {min }}$ and maximum $a_{\text {max }}$ cutoffs. We adopted $q=3.5$ for the power index of the particle distribution and $a_{\max }=1000 \mu \mathrm{m}$ for all the models. The minimum grain size is set to be close to the radiation blowout size estimated based on the grain density and stellar luminosity and mass. We assumed that the debris is uniformly distributed (constant surface density) from the inner radius $\left(R_{i n}\right)$ to the outer radius $\left(R_{\text {out }}\right)$, and computed the thermal dust emission under optically thin conditions where the star is the only heating source. We then derived the best-fit inner and outer boundaries of the cold disk component, along with the total cold disk dust mass, quantifying the goodness of fit with reduced $\chi^{2}$. As detailed in the following sections, we excluded any long wavelength data which was contaminated by nearby background galaxies, as well as any short wavelength data which might include a contribution from a warm disk component.

In some cases, weak warm excess shortward of $\sim 20 \mu \mathrm{m}$ was present. When the warm excess signal was greater than the uncertainty in photospheric subtraction, we also performed a blackbody fit to the short wavelength data to derive the warm component's approximate temperature. Because the location of the tentative warm disks ( $\lesssim 2$ to $10 \mathrm{AU})$ is less than the inner working angle of our high contrast observations, we do not perform detailed SED modeling for the warm excesses. We comment on the derived warm dust temperatures, but their nature will be discussed in a separate paper.

\section{RESULTS AND DISCUSSION}

The disk sizes ( $R_{\text {in }}$ and $R_{\text {out }}$ ) estimated from our simple SED modeling are only meant to provide a rough comparison between the expected disk location and the point-source detection contrast curves presented in Section 4. Some disks we modeled appear to have a very wide dust spatial distribution, and this is probably because we fit the SED with only one component. It is possible that such a cold disk SED (with a wide dust distribution) is composed of two different populations (warm/cold belts+disk halo) like the HD 95086 system (Su et al. 2014). Furthermore, the SED from a typical debris disk, where the sizes of dust particles are in a steep power-law form, is dominated by small grains (less than a few times the blowout size) that are sensitive to non-gravitational forces. The fine debris distribution is expected to be broader than that of their parent bodies.

Three disks (HD 17848, HD 134888, and HD 28355) show signs of warm excesses in their disk SEDs (see Figure 1). In two cases (HD 17848 and HD 28355), the amount of warm excess is small and dominated by the errors from the photospheric subtraction. The temperatures of the tentative excesses for these two systems were reported in the literature. We report the blackbody fit for the warm component in the remaining system, HD 134888. As the focus of this survey was to search for companions sculpting the cold disk components, we only derived detailed parameters for the cold components.

We summarize the derived disk properties: cold disk inner and outer radii, and dust mass (grains up to $1 \mathrm{~mm}$ ) in Table 2. In addition to the cold disk properties, Table 2 also lists the distance and $L^{\prime}$ mag for our sources ( $L^{\prime}$ mag converted from the 2MASS survey Cutri et al. 2003 following Cox 2000). The disk properties for HD 110058 are not calculated due to the lack of sky coverage. The distances are extracted from the parallax (van Leeuwen 2007).

Figure 2 shows the resulting $5 \sigma$ contrast curves for each of the targets. The dotted line is the edge of the full FOV coverage, beyond this line we have reduced coverage (see Figure 3 ). The hashed region is the mean value of the inner and outer edge of the cold debris belt, based on our SED modeling. For each target, we adopt the age of the system (discussion below) and convert the contrast limit to planet masses with the COND evolutionary models (Baraffe et al. 2003). On average we are sensitive to planetary mass companions $\left(\lesssim 13 M_{\text {Jup }}\right.$ ) outward of 0 .'5 from the star, with the exception of HD 17848 and HD 28355 which are older than the other targets. HD 134888 and HD 110058 have only one APP hemisphere coverage. HD 134888 achieved $75 \%$ coverage (P.A. $=135^{\circ}$ to $-135^{\circ}$, clock-wise passing through North) around the star due to large sky rotation. HD 110058 did not have large sky rotation (P.A. $=100^{\circ}$ to $-90^{\circ}$, clock-wise passing through North), thus while the contrast curve is shown, we do not consider this target fully observed. Below we discuss each of the targets individually. 

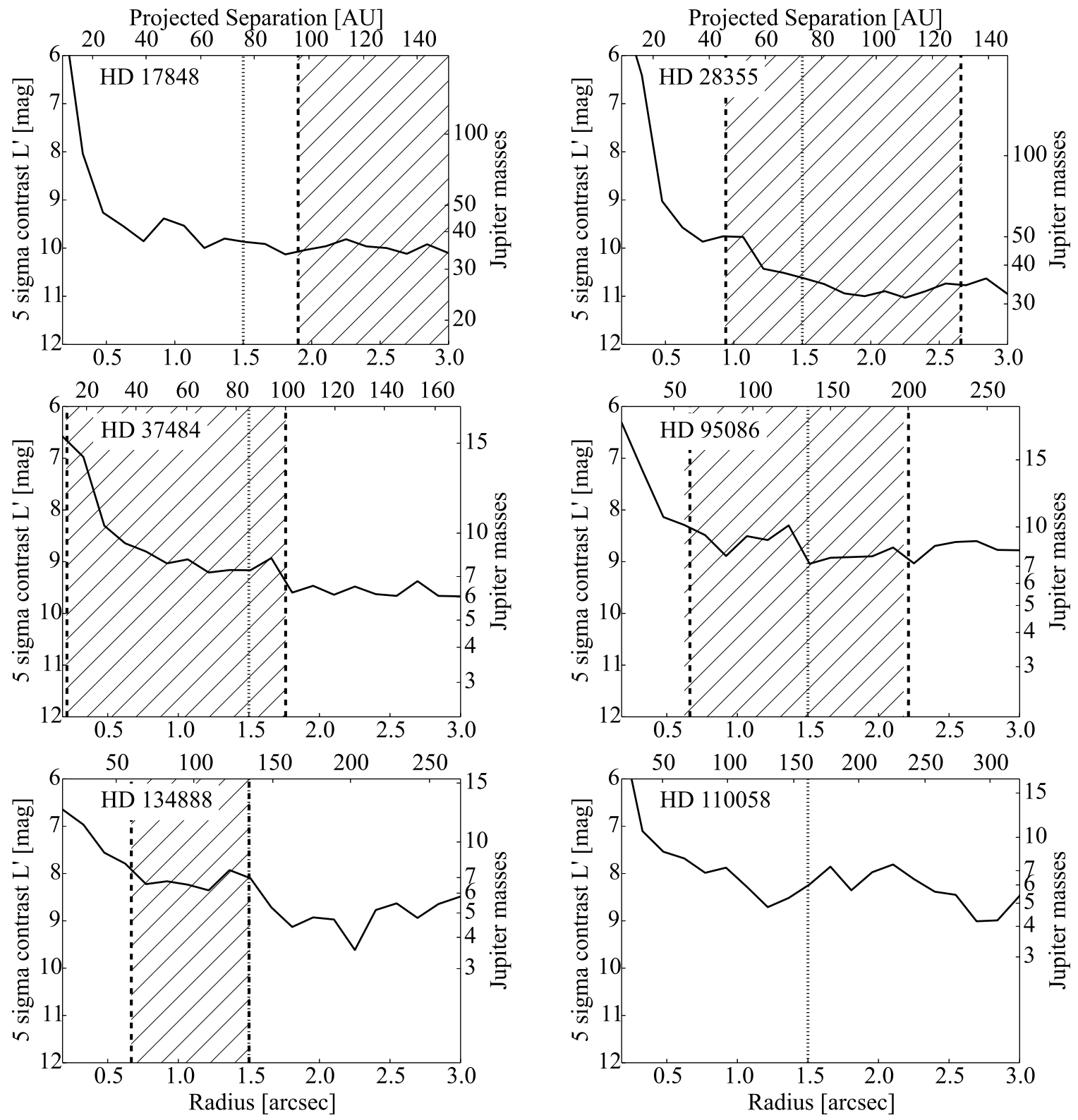

Figure 2. Sensitivity curves for the targets. Each target has the $5 \sigma$ contrast curve in mag and Jupiter masses (based on the COND evolutionary tracks Baraffe et al. 2003) versus separation in arcseconds and AU. The vertical dotted line indicates the edge of the full FOV coverage ( 1 ". 5 ). The hashed region marks the mean value of the inner and outer edge of the cold disk based on our disk modeling. HD 134888 and HD 110058, have only one APP hemisphere coverage. HD 134888 has $270^{\circ}$ coverage due to large sky rotation. The contrast axis (left) and radius in arcsec axis (bottom) is fixed relative to each figure for comparison.

\subsection{HD17848}

HD 17848 ( $v$ Hor) is an A2V (Houk \& Cowley 1975) field star at distance $d=50.5 \pm 0.5 \mathrm{pc}$ (adopting parallax $\varpi=19.82 \pm 0.18$ mas from van Leeuwen 2007). We estimate the effective temperature to be $T_{\text {eff }}=8470 \pm 130 \mathrm{~K}$ based on multiple photometric $T_{\text {eff }}$ estimates: using $U B V K_{s}$ photometry with the color $/ T_{\text {eff }}$ table of Pecaut \& Mamajek (2013), and the tight cluster of reported $T_{\text {eff }}$ estimates in the literature (Allende Prieto \& Lambert 1999; Paunzen et al. 2006; Rhee et al. 2007; Patel et al. 2014). Our adopted $T_{\text {eff }}$ is systematically cooler than that reported by Chen et al. (2014) $(9000 \mathrm{~K})$, which is an outlier compared to the other estimates. Adopting the $\mathrm{V}$ magnitude from Mermilliod (1994) $(V=5.254 \pm 0.005)$, van Leeuwen (2007) parallax, and adopted $T_{\text {eff-appropriate bolo- }}$ metric correction $\mathrm{BC}_{V}=-0.040 \pm 0.034$ (Pecaut \& Mamajek
2013), we estimate $v$ Hor's luminosity to be $\log \left(\mathrm{L} / L_{\odot}\right)=$ $1.222 \pm 0.016$ dex. Based on our HR diagram position for $v$ Hor, we use the evolutionary tracks of Bertelli et al. (2009) to infer an isochronal age of $\sim 540 \mathrm{Myr}$ and mass $1.90 M_{\odot}$. Sampling a reasonable range of protostellar chemical compositions $(Y=0.26-0.27, Z=0.014-0.017)$, we estimate isochronal age $1 \sigma$ uncertainties of $\pm 90 \mathrm{Myr}$ (statistical) and $\pm 60 \mathrm{Myr}$ (systematic), and mass $1 \sigma$ uncertainties of $\pm 0.02 M_{\odot}$ (statistical) and $\pm 0.04 M_{\odot}$ (systematic). Hence, we do not find support for the young age of $\sim 100 \mathrm{Myr}$ proposed by Rhee et al. (2007), and estimate $v$ Hor to be $\sim 5 \times$ older than previously thought.

Its IRS spectrum was first analyzed by Ballering et al. (2013), and suggested the system is a two-component disk with dust temperatures of $164 \mathrm{~K}$ and $50 \mathrm{~K}$. However, Chen et al. (2014) suggested a much warmer temperature, $353_{-8}^{+4} \mathrm{~K}$, while the cold temperature $(57 \pm 5 \mathrm{~K})$ was consistent with the early result. 


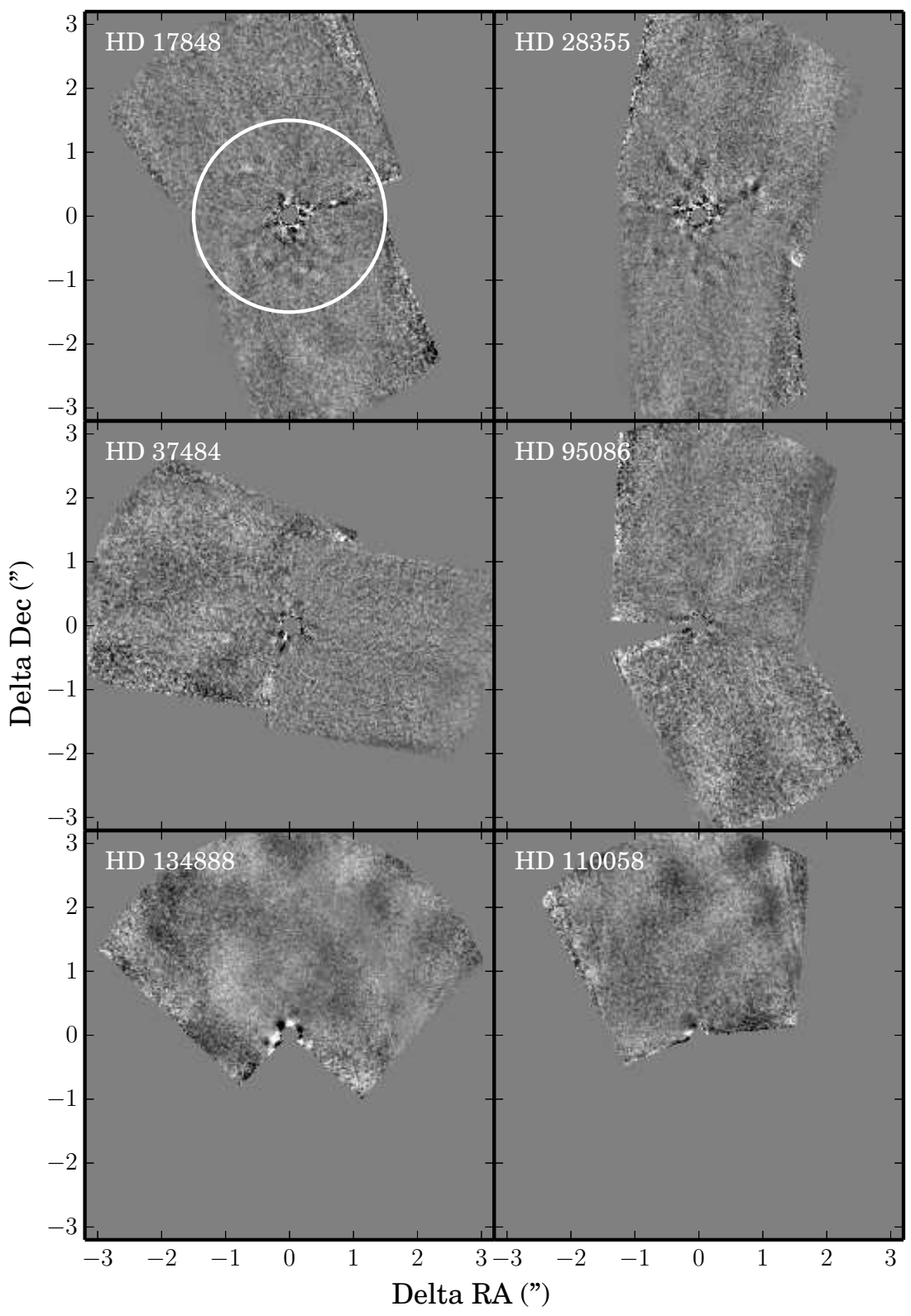

Figure 3. Final PCA reduced images of all six of our targets generated with optimized PCA. The full sensitivity FOV is indicated by the white circle in HD 17848 at 1".5. Beyond 1".5 we do not have full coverage because we only observe on the upper quartile portion of the CCD. The color range in each of the images is limited to $\pm 5 \sigma$. The point source around HD 17848 is an artifact.

The discrepancy is most likely due to how the excess flux in the mid-infrared range was determined (especially how the different modules of IRS spectra joined and pinned down to the photosphere). Therefore, the nature and amount of warm excess is still in debate. Using the observed dust temperature as gauges, the warm excess characterized by Ballering et al. (2013) would be asteroid-like with orbital distances of 9-12 AU, while the value from Chen et al. (2014) would be terrestrial-like with orbital distances of 2-3 AU. The new far-infrared photometry from Herschel and the MIPS-SED data confirm that the cold disk has a typical dust temperature of $\sim 56 \mathrm{~K}$ (see Figure 1). We excluded data points shortward of $\sim 30 \mu \mathrm{m}$ in our SED model, resulting in an inner cold disk radius of $96_{-37}^{+9} \mathrm{AU}$ and an outer cold radius of $410_{-96}^{+24} \mathrm{AU}$ with a total dust mass of $(1.3 \pm 0.7) \times 10^{-3} M_{\oplus}$.

No bright point sources were detected around HD 17848 (see Figure 3). The overlapping region between two APP hemispheres is noisy due to the edge of the dark APP hole. Therefore, the bright point (at 0.7 and P.A. $=-74^{\circ}$ ) on the reduced image is probably a noise spike. We compared any possible faint point sources in our data with archival data obtained with $\mathrm{NaCo} / \mathrm{VLT}$ from both 2011 (087.C-0142(A)) and 2009 (084.C-0396(A)) and verified that none of these faint point sources are present in all three data sets.

Based on the disk structure, we would expect to find companions inside $1^{\prime \prime} .9$ if the inner edge of the cold disk is maintained by unseen planet(s). Our high-contrast observations rule out the presence of a low-mass star down to the brown dwarf regime in the range of $0{ }^{\prime} 5-3^{\prime \prime}(\sim 25-150 \mathrm{AU})$. Bear in mind, as shown in Figure 3, beyond 1".5 we do not have complete sky coverage around the star. As mentioned in Section 3.1, the HD 17848 disk may be an edge-on. This limits our ability to detect companions unless they are fortuitously at a projected separation outward of 0.5 . 


\subsection{HD 28355}

HD 28355 is an A7V (Abt \& Morrell 1995) member of the 650 Myr-old Hyades cluster (Su et al. 2006; De Gennaro et al. 2009) at a distance of $48.8 \pm 0.6 \mathrm{pc}$ away (van Leeuwen 2007). Its IRS spectrum along with MIPS $70 \mu \mathrm{m}$ photometry has been published by several papers with different derived dust temperatures. Morales et al. (2011) suggest the disk SED is best described by two temperatures of $128 \mathrm{~K}$ and $60 \mathrm{~K}$, which are consistent with the values $(120 \mathrm{~K}+54 \mathrm{~K})$ published by Ballering et al. (2013) within errors. Analysis performed by Chen et al. (2014) gives the warm temperature of $176_{-8}^{+7} \mathrm{~K}$ and the cold temperature of $69_{-6}^{+5} \mathrm{~K}$. As discussed in Section 3, HD 28355 is near a bright infrared source, which is extended at $160 \mu \mathrm{m}$, as a result its far-infrared fluxes are contaminated. The overall disk SED can be fit with two temperatures: $\sim 120 \mathrm{~K}$ and $\sim 55 \mathrm{~K}$ (see Figure 1). However, the contamination-free part of the SED (data shortward of $\sim 80 \mu \mathrm{m}$ ) can also be described by one single temperature of $\sim 80 \mathrm{~K}$ (within a few $\sigma$ ). Therefore, we excluded the Herschel $160 \mu \mathrm{m}$ data and the MIPS-SED points longward of $85 \mu \mathrm{m}$ in our one-component SED model. The inner boundary is estimated to be $46 \pm 12 \mathrm{AU}$, and the outer is $130 \pm 30 \mathrm{AU}$ with a total dust mass of $(1.8 \pm 0.7) \times 10^{-3} M_{\oplus}$.

No bright point sources were detected around HD 28355 (see Figure 3). The structure inward of $1^{\prime \prime} .5$ is due to residuals from the APP PSF. We were not sensitive to planetary mass companions due to the older age of the star. We were able to rule out substellar companions from 0.'5-3" ( 24-147 AU). Our single component SED model places the inner boundary of the disk at $0{ }^{\prime} 9$. Thus, if the cold disk is shaped by a companion, it must be less than $\sim 50 M_{\text {Jup }}$.

\subsection{HD 37484}

HD 37484 is a F3V (Houk 1982) star at a distance of $d=56.8 \pm 2.0 \mathrm{pc}$ (adopting parallax $\varpi=17.61 \pm 0.62 \mathrm{mas}$ from van Leeuwen 2007). Its $B-V$ versus $M_{V}$ position places it on the zero age main sequence (ZAMS), thus it must be $>27 \mathrm{Myr}$ in order to allow enough time for a $\sim 1.36 M_{\odot}$ star to reach the main sequence. Its position is commensurate with that of the Pleiades and IC2391 clusters, making it $<100 \mathrm{Myr}$. It has a brighter and hotter $(\sim \mathrm{A} 5 \mathrm{~V})$ common proper motion companion 0.34 deg away (HR 1915). Both stars are considered Columba members by Malo et al. (2013), who list 10-40 Myr for the group. The companion appears to be on or below the ZAMS, consistent with an age of >19 Myr. HD 37484's HR diagram position is consistent with the previous assigned age of 30 Myr. Given its Columba membership and consistency with the age of other Columba members, we adopt the age of $30 \pm 10$ Myr.

The disk SED is composed of data from Spitzer MIPS 24 and $70 \mu \mathrm{m}$ photometry as well as IRS $10-35 \mu \mathrm{m}$ spectroscopy. The disk SED is best fit with a blackbody temperature of $\sim 88 \mathrm{~K}$. The best fitting disk model has an inner radius of $12_{-4}^{+20} \mathrm{AU}$, outer radius of $100_{-20}^{+100} \mathrm{AU}$ and dust mass of $(2 \pm 1) \times 10^{-3} M_{\oplus}$. This source does not have longer wavelength Herschel data, which makes the outer disk radius and dust mass fairly unconstrained.

We initially detected a faint point source around HD 37484 at 0.97 (55 AU from the star), 9.0 magnitudes fainter than the host star at a P.A. of $103^{\circ}$ in our APP data (see Figure 3). However, this point source was not detected in archival data with equivalent sensitivity taken in 2011 (088.C-0085(A)), allowing us to conclude that it is unlikely to be a real source. This false detection demonstrates the importance of archival data, which can be used to quickly confirm or deny the physical nature of a point source.

\subsection{HD 95086}

HD 95086 is a 17 Myr old A8 LCC member star at a distance of $90.4 \pm 3.3 \mathrm{pc}$ (Meshkat et al. 2013). The system was also resolved by Herschel at 70 and $100 \mu \mathrm{m}$ (Moór et al. 2013) with an estimated inclination of $25^{\circ}$ from face-on. Re-analysis of the Herschel resolved images combined with detailed SED modeling reveal that the extended part of the images arises from a disk halo ( $\mathrm{Su}$ et al. 2014), similar to the disk halo found around HR 8799 (Su et al. 2009; Matthews et al. 2014). In the three-component disk model presented by Su et al. (2014), the inner belt is located from $\sim 7 \mathrm{AU}$ to $\sim 10 \mathrm{AU}$, and the cold planetesimal disk likely ranges from $63 \pm 6 \mathrm{AU}$ to $189 \pm 13 \mathrm{AU}$, and are surrounded by an extended halo up to $\sim 800 \mathrm{AU}$.

A $5 M_{\text {Jup }}$ planet was discovered around HD 95086 by Rameau et al. (2013b). Our $H$-band NICI/Gemini non-detection provided a strict color lower limit for the planet of $H-L^{\prime}>3.1 \pm$ 0.5 mag (Meshkat et al. 2013). The subsequent $H$-band Gemini Planet Imager (GPI) detection of HD $95086 \mathrm{~b}$ by (Galicher et al. 2014) provides a red planet color of $H-L^{\prime}=3.6 \pm 1.0$. We also observed the system with the APP, but did not detect it due to decreased AO quality at high airmass (>1.4) during the observation (APP hemisphere 2; see Figure 3). Due to lack of sky rotation, we are missing about $20^{\circ}$ of coverage. Figure 2 shows that we achieve a sensitivity of $\sim 10 M_{\text {Jup }}$ at the separation of the planet, however the detected planet is $5 \pm 2 M_{\text {Jup }}$ (Rameau et al. 2013a).

\section{5. $H D 134888$}

HD 134888 is an F4V star, located $90_{-8}^{+9}$ pc away (adopting parallax $\varpi=11.12 \pm 1.05$ mas from van Leeuwen 2007). We adopt an age of $16 \mathrm{Myr}$ based on its membership in the Lower Centaurus-Crux (LCC) association, commensurate with isochronal age estimates for the star (16-25 Myr; Pecaut et al. 2012). Chen et al. (2014) conclude it has a two-component disk with the warm temperature of $387_{-7}^{+6} \mathrm{~K}$ and the cold temperature of $72 \pm 5 \mathrm{~K}$. The far-IR excesses along with the IRS excess longward of $\sim 30 \mu \mathrm{m}$ are consistent with a blackbody temperature of $75 \mathrm{~K}$ (see Figure 1). However, we found the warm excess is better fit with a dust temperature of $\sim 175 \mathrm{~K}$ (i.e., asteroid-like). The derived temperature in the warm component is highly dependent on the amount and shape of the excess emission in the $10-20 \mu \mathrm{m}$ region. Future mid-IR observations are needed to better characterize the warm component. We excluded data points shortward of $\sim 30 \mu \mathrm{m}$ in our SED fitting of the cold component. The best-fit one cold-component disk ranges from $60 \pm 11 \mathrm{AU}$ to $135 \pm 29 \mathrm{AU}$ with a dust mass of $3.25 \pm 1.4 \times 10^{-2} M_{\oplus}$.

Based on our inferred disk structure, the ideal place for detecting the potential low-mass companion that sculpts the disk is interior of $60 \mathrm{AU}\left(0^{\prime \prime} 66\right)$ or exterior of $135 \mathrm{AU}\left(1^{\prime \prime} .5\right)$. A possible interesting point source appeared at 0.2 from the star, but after comparing with archival data (087.C-0142(A)), the point source appears to be an artifact of the data reduction. No believable point sources are detected in our reduced images around the star at any projected separation, however we cannot conclusively say there are no companions around HD 134888. From a P.A. of $135^{\circ}$ to $-135^{\circ}$ (clock-wise, passing through North; see Figure 3), we do not detect any companions with an average mass limit of 5-7 $M_{\text {Jup }}$. 


\section{6. $H D 110058$}

HD 110058 is a A0V star (Houk 1978) and member of the LCC subgroup of Sco-Cen (de Zeeuw et al. 1999; Rizzuto et al. 2011). The revised Hipparcos parallax from van Leeuwen (2007) ( $\varpi=9.31 \pm 0.78$ mas) translates to a distance of $107_{-8}^{+10} \mathrm{pc}$. The star is situated near $\ell, b=301^{\circ},+13.6$, in the northern part of LCC, which appears to be the oldest part of the subgroup (Preibisch \& Mamajek 2008; Pecaut et al. 2012), so we follow Chen et al. (2014) and adopt an age of $17 \mathrm{Myr}$.

We did not achieve full $360^{\circ}$ APP coverage around this star. Only one APP hemisphere was observed and it had poor sky rotation. Without full sky coverage, the contrast curve for HD 110058 (Figure 2) is only valid from a P.A. of $\sim 100^{\circ}$ to $-90^{\circ}$ (clock-wise, passing through North; see Figure 3). We are sensitive to 5-8 $M_{\text {Jup }}$ from $0.5-3^{\prime \prime}$. We detect no point sources in this limited region around the star.

\section{CONCLUSIONS}

We present the first results from our survey searching for planets around stars with bright debris disks with gaps. In this paper, we present the data from six targets obtained with the APP coronagraph on $\mathrm{NaCo} / \mathrm{VLT}$. One of our targets, HD 95086, was found to harbor a planet on the inner edge of the outer debris belt (Rameau et al. 2013b; Moór et al. 2013; Galicher et al. 2014). While our data were not sensitive enough to detect this planet (Meshkat et al. 2013), its discovery demonstrates the strength of two-component disk targets.

For each of the targets, we derive disk properties based on our SED models. These properties (including disk mass and debris radial distribution) allow us to infer the likely location of gaps in the disk, which may be carved out by planets. We detect no companions in our APP data. A few plausible point sources were detected but ruled out after comparison with archival data. We were sensitive to planetary-mass companions for four of the six targets, using COND atmosphere models. If cool planets have substantial methane absorption and little cloud opacity, as is predicted by the COND evolutionary models (Baraffe et al. 2003), then $L^{\prime}$-band will be less sensitive to planets. However, the majority of directly imaged planets do not behave like field brown dwarfs of similar effective temperature. Most cool planets do not show evidence of methane absorption (e.g., HR8799bcd and 2M1207b Skemer et al. 2014) and are redder than predicted ( $H-L^{\prime}=2$ to 3 mag: Galicher et al. 2014) Thus, our use of $L^{\prime}$-band is comparable to the best $H$-band surveys at separations where we are contrast limited. The benefit of the APP for companion discovery over direct imaging is inconclusive, based on our sample of six observations. See T. Meshkat et al. (in preparation) for further discussion.

The sample size of six targets is too small to draw conclusions about the origins of the gaps in Holey Debris Disks. Our complete Holey Debris Disks sample (V. Bailey et al. in preparation) will allow stronger statements of whether the gaps in these disks are formed by one massive companion, multiple low-mass companions, or other mechanisms. We discuss each target individually and analyze the sensitivity of companions achieved relative to the boundaries of the debris disks, based on our disk models.

In order to detect lower mass planets at the inner edges of the cold outer debris belts, greater sensitivity needs to be achieved. In this paper, we have only modeled the outer single cold component, however many of our targets may be twocomponent disks. The projected separation of the warm inner disk components are much less than the inner working angle limit of current high-contrast imaging data, and so planets sculpting the warm disks would remain undetected. Discoveries like that of the low-mass planet HD $95086 \mathrm{~b}$ strengthen the notion that gaps need not be carved by a single very massive companions (Dodson-Robinson \& Salyk 2011), and thus future surveys will require increased sensitivity in addition to smaller inner working angle.

We thank the anonymous referee for suggestions that improved this paper. We thank S. Quanz for assistance in acquiring these data. T.M. and M.A.K. acknowledge funding under the Marie Curie International Reintegration Grant 277116 submitted under the Call FP7-PEOPLE-2010-RG. V.B. acknowledges support from the NSF Graduate Research Fellowship Program (DGE-1143953). K.Y.L.S. is partially supported by NASA grant NNX11AF73G. E.E.M. acknowledges support from NSF award AST-1313029. This paper made use of the SIMBAD and VIZIER databases.

\section{REFERENCES}

Abt, H. A., \& Morrell, N. I. 1995, ApJS, 99, 135

Allende Prieto, C., \& Lambert, D. L. 1999, A\&A, 352, 555

Bailey, V., Hinz, P. M., Currie, T., et al. 2013, ApJ, 767, 31

Ballering, N. P., Rieke, G. H., Su, K. Y. L., \& Montiel, E. 2013, ApJ, 775, 55

Balog, Z., Müller, T., Nielbock, M., et al. 2014, ExA, 37, 129

Baraffe, I., Chabrier, G., Barman, T. S., Allard, F., \& Hauschildt, P. H. 2003, A\&A, 402, 701

Bertelli, G., Nasi, E., Girardi, L., \& Marigo, P. 2009, A\&A, 508, 355

Biller, B. A., Liu, M. C., Wahhaj, Z., et al. 2013, ApJ, 777, 160

Biller, B. A., Males, J., Rodigas, T., et al. 2014, ApJL, 792, L22

Brandt, T. D., McElwain, M. W., Turner, E. L., et al. 2014, ApJ, 794, 159

Castelli, F., \& Kurucz, R. L. 2004, arXiv:astro-ph/0405087

Chauvin, G., Vigan, A., Bonnefoy, M., et al. 2014, e-print (arXiv:1405.1560)

Chen, C. H., Mittal, T., Kuchner, M., et al. 2014, ApJS, 211, 25

Chun, M., Toomey, D., Wahhaj, Z., et al. 2008, Proc. SPIE, 7015, 1

Cox, A. N. 2000, Allen's astrophysical quantities (4th ed.; New York: AIP Press; Springer)

Cutri, R. M., Skrutskie, M. F., van Dyk, S., et al. 2003, yCat, 2246, 0

De Gennaro, S., von Hippel, T., Jefferys, W. H., et al. 2009, ApJ, 696, 12

de Zeeuw, P. T., Hoogerwerf, R., de Bruijne, J. H. J., Brown, A. G. A., \& Blaauw, A. 1999, AJ, 117,354

Desidera, S., Covino, E., Messina, S., et al. 2014, e-print (arXiv:1405.1559)

Dodson-Robinson, S. E., \& Salyk, C. 2011, ApJ, 738, 131

Galicher, R., Rameau, J., Bonnefoy, M., et al. 2014, A\&A, 565, L4

Houk, N. 1978, Michigan catalogue of two-dimensional spectral types for the HD stars (Ann Arbor, MI: Univ. of Michigan)

Houk, N. 1982, Michigan Catalogue of Two-dimensional Spectral Types for the HD stars. Volume 3. Declinations -40_0 to -26_0 (4th ed.; New York: AIP Press; Springer)

Houk, N., \& Cowley, A. P. 1975, University of Michigan Catalogue of twodimensional spectral types for the HD stars (4th ed.; New York: AIP Press; Springer)

Janson, M., Brandt, T. D., Moro-Martín, A., et al. 2013, ApJ, 773, 73

Kenworthy, M., Quanz, S., Meyer, M., et al. 2010, Msngr, 141, 2 Kenworthy, M. A., Meshkat, T., Quanz, S. P., et al. 2013, ApJ, 764, 7 Kuzuhara, M., Tamura, M., Kudo, T., et al. 2013, ApJ, 774, 11 Lafrenière, D., Doyon, R., Marois, C., et al. 2007, ApJ, 670, 1367 Lebouteiller, V., Barry, D. J., Spoon, H. W. W., et al. 2011, ApJS, 196, 8 Leisenring, J. M., Skrutskie, M. F., Hinz, P. M., et al. 2012, Proc. SPIE, 8446, 4 Lenzen, R., Hartung, M., Brandner, W., et al. 2003, Proc. SPIE, 4841, 944 Lu, N., Smith, P. S., Engelbracht, C. W., et al. 2008, PASP, 120, 328 Malo, L., Doyon, R., Lafrenière, D., et al. 2013, ApJ, 762, 88

Marois, C., Doyon, R., Racine, R., \& Nadeau, D. 2000, PASP, 112, 91 Marois, C., Lafrenière, D., Doyon, R., Macintosh, B., \& Nadeau, D. 2006, ApJ, 641,556

Marois, C., Macintosh, B., Barman, T., et al. 2008, Sci, 322, 1348

Marois, C., Macintosh, B., \& Véran, J.-P. 2010, Proc. SPIE, 7736, 1

Matthews, B., Kennedy, G., Sibthorpe, B., et al. 2014, ApJ, 780, 97

Mermilliod, J.-C. 1994, BICDS, 45, 3

Meshkat, T., Bailey, V., Rameau, J., et al. 2013, ApJL, 775, L40 
Meshkat, T., Kenworthy, M. A., Quanz, S. P., \& Amara, A. 2014, ApJ, 780, 17 Moór, A., Ábrahám, P., Kóspál, Á., et al. 2013, ApJL, 775, L51

Morales, F. Y., Rieke, G. H., Werner, M. W., et al. 2011, ApJL, 730, L29

Nielsen, E. L., Liu, M. C., Wahhaj, Z., et al. 2013, ApJ, 776, 4

Patel, R. I., Metchev, S. A., \& Heinze, A. 2014, ApJS, 212, 10

Paunzen, E., Schnell, A., \& Maitzen, H. M. 2006, A\&A, 458, 293

Pawellek, N., Krivov, A. V., Marshall, J. P., et al. 2014, ApJ, 792, 65

Pecaut, M. J., \& Mamajek, E. E. 2013, ApJS, 208, 9

Pecaut, M. J., Mamajek, E. E., \& Bubar, E. J. 2012, ApJ, 746, 154

Preibisch, T., \& Mamajek, E. 2008, in The Nearest OB Association: ScorpiusCentaurus (Sco OB2), ed. B. Reipurth (San Francisco, CA: ASP), 235

Quanz, S. P., Meyer, M. R., Kenworthy, M. A., et al. 2010, ApJL, 722, L49

Quillen, A. C. 2006, MNRAS, 372, L14

Quillen, A. C., \& Thorndike, S. 2002, ApJL, 578, L149

Rameau, J., Chauvin, G., Lagrange, A.-M., et al. 2013a, ApJL, 779, L26

Rameau, J., Chauvin, G., Lagrange, A.-M., et al. 2013b, ApJL, 772, L15

Reggiani, M., Quanz, S. P., Meyer, M. R., et al. 2014, ApJL, 792, L23

Rhee, J. H., Song, I., Zuckerman, B., \& McElwain, M. 2007, ApJ, 660, 1556
Rizzuto, A. C., Ireland, M. J., \& Robertson, J. G. 2011, MNRAS, 416, 3108

Rousset, G., Lacombe, F., Puget, P., et al. 2003, Proc. SPIE, 4839, 140

Sierchio, J. M., Rieke, G. H., Su, K. Y. L., \& Gáspár, A. 2014, ApJ, 785, 33

Sivanandam, S., Hinz, P. M., Heinze, A. N., Freed, M., \& Breuninger, A. H. 2006, Proc. SPIE, 6269, 0

Skemer, A. J., Marley, M. S., Hinz, P. M., et al. 2014, ApJ, 792, 17

Skrutskie, M. F., Jones, T., Hinz, P., et al. 2010, Proc. SPIE, 7735, 3

Su, K. Y. L., \& Rieke, G. H. 2014, in IAU Symp. 299, Exploring the Formation and Evolution of Planetary Systems, ed. M. Booth, B. C. Matthews, \& J. R. Graham (Cambridge: Cambridge Univ. Press), 318

Su, K. Y. L., Rieke, G. H., Stansberry, J. A., et al. 2006, ApJ, 653, 675

Su, K. Y. L., Rieke, G. H., Stapelfeldt, K. R., et al. 2009, ApJ, 705, 314

Su, K. Y. L., Rieke, G. H., Stapelfeldt, K. R., et al. 2010, American Astronomical Society Meeting Abstracts \#215, 42, 428.26

Su, K. Y. L., Morrison, S., Malhotra, R., et al. 2014, ApJ, in press (arXiv:1402.0167)

van Leeuwen, F. 2007, A\&A, 474, 653

Vigan, A., Patience, J., Marois, C., et al. 2012, A\&A, 544, A9

Wahhaj, Z., Liu, M. C., Nielsen, E. L., et al. 2013, ApJ, 773, 179 